\title{
Predication of 3-D Viscous Flowfield of a Centrifugal Impeller
}

\author{
Limin Gao, Xudong Feng, Jian Xie \\ School of Power and Energy, Northwestern Polytechnical University, Xi'an, China \\ E-mail:gaolm@nwpu.edu.cn \\ Received September 10, 2010; revised November 5, 2010; accepted November 15, 2010
}

\begin{abstract}
A three-dimensional viscous code has been developed to solve Reynolds-averaged Navier-Stokes equations. The governing equations in finite volume form are solved by two-step Runge-Kutta scheme with implicit residual smoothing. The eddy viscous is obtained using the Baldwin-Lomax model. A prediction of the 3-D turbulent flow and the performance in the "all-over controlled vortex distribution" centrifugal impeller with a vaneless diffuser has been made for the compressor at design and off-design condition. The predicted efficiency is a little higher than the experiment data. These results suggest that the present calculation code is able to determine the flow development in the impeller and also the turbulence model in the centrifugal impeller should be improved.
\end{abstract}

Keywords: Centrifugal Impeller, Aerodynamic Performance, 3-D Viscous Flow Calculation, Design \& off-Design Conditions

\section{Introduction}

Centrifugal compressors are used widely in industry due to their advantages of simple structure and high-pressure ratio. However, their efficiency and stability are adversely influenced by the present of impeller exit flow non-uniformity. In recent years, as a result of improvements in experimental techniques and numerical methods, it has been possible to avoid the non-uniformity of exit flow field in order to obtain improved efficiency and stability of an impeller. The work of Eckardt's [1] and Krain's [2] are most representative in all related experimental research. Their studies indicated that both complicated secondary flows and separated boundary layer would cause the radial and circumferential non-uniform flows at the outlet, and consequently the performance of the centrifugal compressor decreases. Meanwhile, some numerical codes have also been developed to study the flowfield in centrifugal compressor by other researchers. Most of them [2-4] have provided the detailed flowfield to understand the complicated flow. However, CFD is still a little immature and does not reach the engineering implementation level. Further research is necessary to develop a more accurate and faster, numerical predicting code, which will provide the sophisticated tool to predict the aerodynamic performance of a high-speed centrifugal compressor for designing an impeller with higher pressure-ratio and efficiency.

In the present work, a 3-D turbulent code has been developed, and the prediction of 3-D viscous flowfield of an AOCV centrifugal impeller has been carried out. Therefore, it is hoped that the present study will provide a useful predication tool to aid in the future experimental work and the industrial design.

\section{AOCV Centrifugal Impeller}

The air compressor, which is applied in a large-scale air-separation plant, is produced from SER Turbomachinery Research Center of the Xi'an JiaoTong University. Its impeller is a three-dimensional centrifugal impeller which is design using the All-Over Controlled Vortex Distribution designing theory.

The impeller is shrouded and has 19 full blades with an exit backswept of 50 degs. The inlet diameter is 0.222 $\mathrm{m}$ and the inlet blade height is $0.0606 \mathrm{~m}$. The exit diameter is $0.340 \mathrm{~m}$ and the exit blade height is $0.0272 \mathrm{~m}$. The design speed of $16360 \mathrm{rpm}$ gives a rotor exit tip speed of $290 \mathrm{~m} / \mathrm{s}$. The design mass flow rate is $2.8 \mathrm{~m}^{3} / \mathrm{s}$ at the standard inlet conditions of $101,325 \mathrm{~N} / \mathrm{m}^{2}$ and $288.15 \mathrm{~K}$. The rotor tip Reynolds number (U2D2/v) is $1.42 \times 10^{6}$. The impeller is showed in Figure 1. 


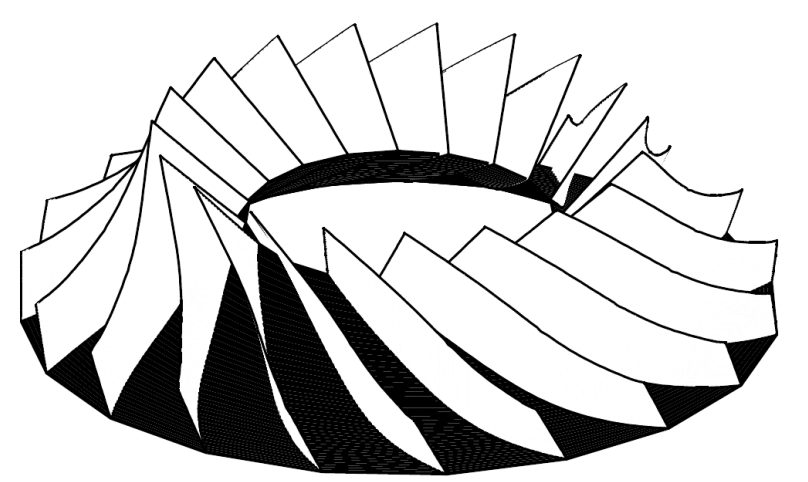

Figure 1. AOCV Impeller.

\section{Computational Method}

\subsection{Governing Equations}

It is convenient to write the three-dimensional Reynolds averaged Navier-Stokes equations in finite volume form cast in the blade-relative frame using cylindrical coordinates $(\mathrm{r}, \theta, \mathrm{x})$ :

$$
\begin{aligned}
& \frac{\partial}{\partial t} \oint \vec{U} d V o l=-\oint\left(\vec{H}_{I}+\vec{H}_{V}\right) \bullet \vec{n} d S+\oint \rho \vec{G}_{I} d V o l \\
& \vec{U}=\left[\begin{array}{l}
\rho \\
\rho W_{z} \\
r \rho W_{\theta} \\
\rho W_{r} \\
\rho E
\end{array}\right] \vec{H}_{I}=\left[\begin{array}{l}
\rho \vec{W} \\
\rho W_{z} \vec{W}+p \vec{e}_{z} \\
r \rho W_{\theta} \vec{W}+r p \vec{e}_{\theta} \\
\rho W_{r} \vec{W}+p \vec{e}_{r} \\
\rho I \vec{W}
\end{array}\right] \vec{H}_{V}=\left[\begin{array}{l}
0 \\
-\tilde{\sigma} \vec{e}_{z} \\
-r \tilde{\sigma} \vec{e}_{\theta} \\
-\tilde{\sigma} \vec{e}_{r} \\
-\tilde{\sigma} \vec{W}
\end{array}\right] \\
& \vec{G}_{I}=\left[\begin{array}{l}
0 \\
0 \\
-2 \omega r W_{r} \\
\frac{W_{\theta}^{2}}{r}+r \omega^{2}+2 \omega W_{\theta}+\frac{p-\sigma_{\theta \theta}}{r} \\
\kappa \nabla T+\dot{q}
\end{array}\right]
\end{aligned}
$$

with $q=W_{x} \hat{i}_{x}+W_{\theta} \hat{i}_{\theta}+W_{r} \hat{i}_{r}$, the relative velocity; $\Omega=$ rotation speed; $\tilde{\tau}=$ the stress tensor (containing both the static pressure and the viscous stresses); and $I=C_{p} T_{\text {orel }}-(\Omega r)^{2} / 2$, the rothalpy. The system is closed by an equation of state

$P=\rho(\gamma-1)\left(E-0.5\left(\vec{q} \cdot \vec{q}-(\Omega r)^{2}\right)\right)$ and a mixing length turbulence model patterned after Baldwin-Lomax.

The code solves the N-S equation in an integral conversation form using hexahedral control volumes formed by a simple H-mesh. The integrals in the conservation equations are replaced by discrete summation around the faces of the computational cell, which is expressed as follows:

$$
\begin{aligned}
& \frac{\Delta \vec{U}_{i, j, k}}{\Delta t} \Delta \operatorname{Vol}_{i, j, k} \\
& =-\sum_{\text {cell }}\left(\vec{H}_{I}+\vec{H}_{V}\right) \cdot \Delta \overrightarrow{\text { Area }}+\rho S_{i, j, k} \Delta \operatorname{Vol}_{i, j, k}=\vec{R}(\vec{U})_{i, j, k}
\end{aligned}
$$

$\vec{R}$ in the above Equation (2) is called as the residual on each 3-D grid element $(i, j, k)$. Fluxes through cell faces are calculated by linear interpolation of the density, velocity, etc., between neighbor cell centers. Thus the formal spatial accuracy is second order on the smoothly varying meshes and global conversation is ensured. A combined second and fourth derivative adaptive artificial viscosity model with pressure gradient switching is added to the discretized equations to eliminate spurious "wiggles" and to control shock capturing. The basic algorithm as described by Dawes (1988) [5] is similar to a two-step Runge-Kutta method plus residual smoothing:

$$
\left\{\begin{array}{l}
\Delta \bar{U}^{(1)}=\frac{\Delta t}{\Delta V O L} \cdot \bar{R}_{i j k}^{n} ; \quad \bar{U}^{(1)}=\bar{U}^{n}+\Delta \bar{U}^{(1)} \\
\Delta \bar{U}^{(2)}=\frac{\Delta t}{\Delta V O L} \cdot \bar{R}_{i j k}^{(2)} ; \quad \bar{U}^{(2)}=\bar{U}^{n}+\Delta \bar{U}^{(1)} \\
\bar{U}^{n}=\bar{U}^{(2)}
\end{array}\right.
$$

In order to improve the computational efficiency, the discretized equations are solved using accelerating techniques, such as the local-time stepping and multigrid methods.

\subsection{Boundary Condition}

At inlet, total temperature and total pressure are fixed and either flow angle or absolute swirl velocity held constant depending on whether the relative flow is sonic or supersonic. At outlet the hub static pressure is fixed. The physical periodic condition upstream and downstream of the impeller is imposed. No slip, no flux and adiabatic wall conditions (or wall temperature) are applied on the solid wall. Additionally, the derivative of the pressure normal to the wall surface is set to zero.

\subsection{Grid for Computation}

There are 89987 calculation points with 29 circumferential points, 29 radial points and 107 streamwise points. To capture the boundary layer, the grids are designed clustering near the wall. The inlet for the 3-D flow calculation was $0.1265 \mathrm{~m}$ upstream of the impeller inlet. The exit radius of the vaneless diffuser for the calculation has a ratio of $\mathrm{r} 3 / \mathrm{r} 2=1.294$ to the impeller outlet. The computing grids are showed in Figure 2. 


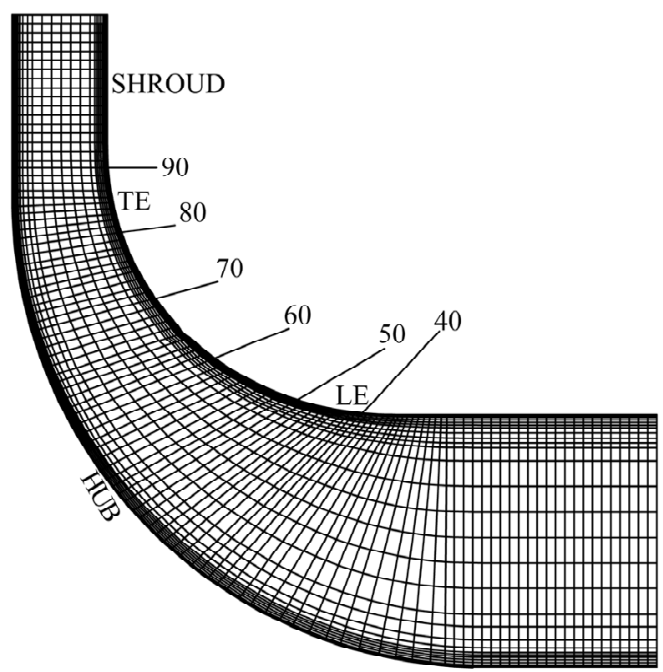

(a)

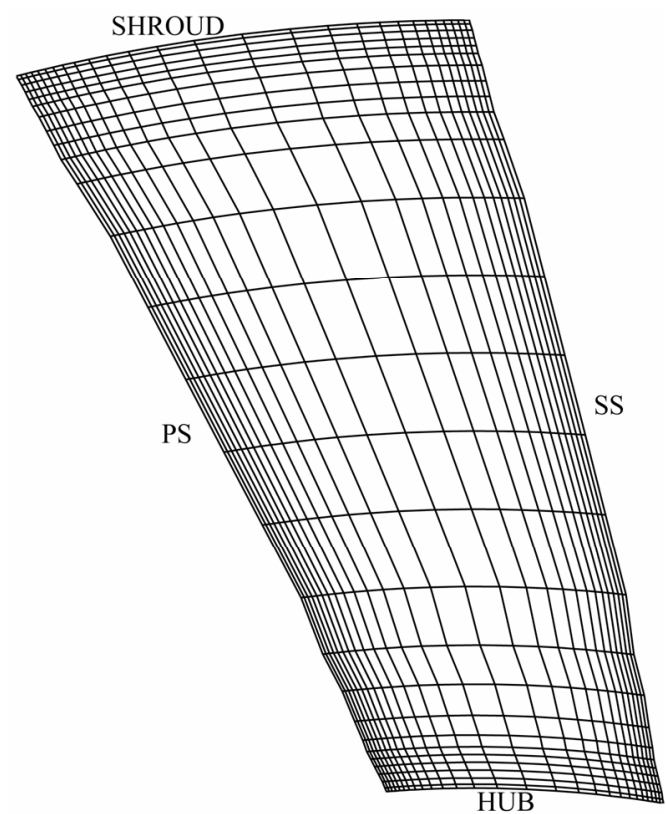

(b)

Figure 2. Grid for 3-D flow computation, (a) Meridional view; (b) Blade-to-blade plane near impeller leading edge.

\subsection{Convergence}

Computing convergence is determined when the follow- ing requirements are satisfied: 1) the mass flow error throughout the domain was deemed acceptable and flow mass in inlet and outlet are in accordance, 2) both RMSM (maximum computing error, which is expressed as Equation (4)) and RMS (summation of computing error, which is expressed as Equation (5)) through all computing points decrease three or four orders; 3 ) the flow variables (velocity or pressure) at the arbitrary computational point is monitored to insure that the velocity field was not changing.

\section{Results of 3-D Prediction}

The predicated meridional velocity vectors on three surfaces with three tangential positions are presented in Figure 2. There is no backflow at the diffuser exit and no separation in the shroud boundary layer all through the compressor. Flow near the pressure side of the impeller passage is shown in Figure 3(a). A secondary flow from the hub to shroud becomes evident in the second half of the passage due to curvature of the passage from the axial to radial direction. At mid-passage, Figure 3(b) shows the shroud boundary layer is thickening in impeller and velocity vectors are nearly parallel to the shroud and the hub. Near the suction side of the impeller, centrifugal and curvature effects combine to give strong secondary flows towards the shroud. This is especially true in the second half of the passage, where flow angles as high as 30 degs to the meridional direction is seen in Figure 3(c). Clearly the meridional velocity near the suction surface is larger than near the pressure surface.

Distribution of the static pressure on the mid-pitch meridional surface through the impeller is presented on the Figure 4. The value on the pressure contours is the ratio of the local static pressure to the inlet total pressure. From the Figure 4, it can be seen that the static pressure is gradually increased and that the distribution of the static pressure is uniform comparatively on the same chord section.

The measured and calculated total/total polytropic impeller efficiency is presented on Figure 5. In the calculation, the centrifugal impeller works on the design and the off-design conditions at 100 percent design speed respectively, totally eight work-conditions. According to

$$
\begin{aligned}
& R M S M=\sqrt{\operatorname{Max}\left(\left(\frac{\Delta \rho}{\Delta t}\right)^{2}+\left(\frac{\Delta \rho V_{z}}{\Delta t}\right)^{2}+\left(\frac{\Delta \rho V_{r}}{\Delta t}\right)^{2}+\left(\frac{\Delta \rho V_{\theta}}{\Delta t}\right)^{2}+\left(\frac{\Delta \rho e}{\Delta t}\right)^{2}\right)_{i, j, k}} \\
& R M S=\sqrt{\frac{\sum_{i, j, k=1}^{I M, J M}\left(\left(\frac{\Delta \rho}{\Delta t}\right)^{2}+\left(\frac{\Delta \rho V_{z}}{\Delta t}\right)^{2}+\left(\frac{\Delta \rho V_{r}}{\Delta t}\right)^{2}+\left(\frac{\Delta \rho V_{\theta}}{\Delta t}\right)^{2}+\left(\frac{\Delta \rho e}{\Delta t}\right)^{2}\right)_{i, j, k}}{I M^{*} J M^{*} K M}}
\end{aligned}
$$




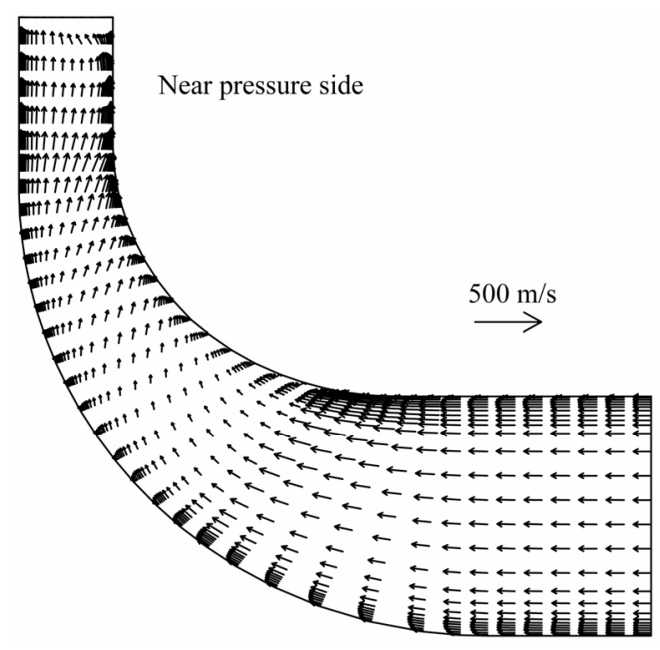

(a)

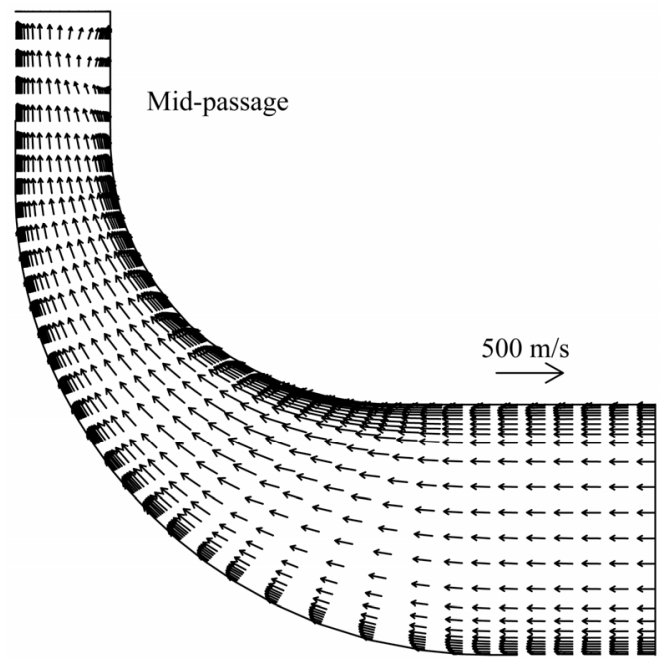

(b)

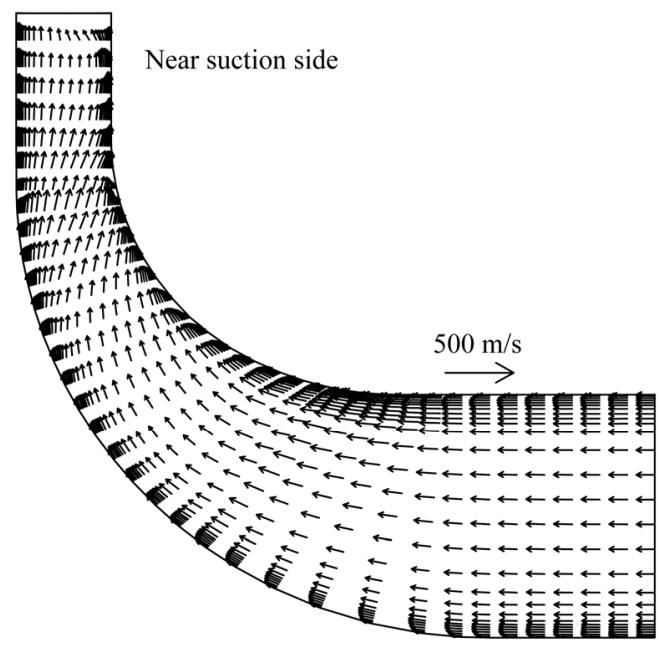

(c)

Figure 3. Meridional views of velocity vectors.

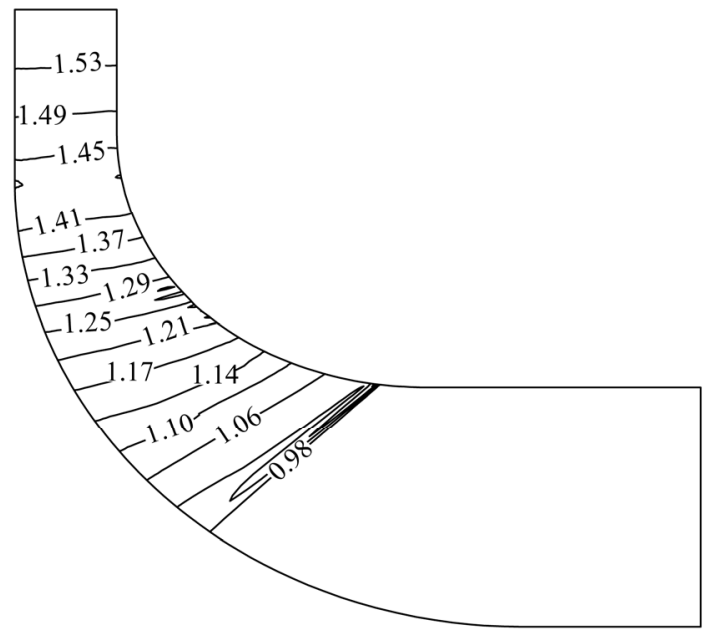

Figure 4. static pressure contour on the mid-pitch surface.

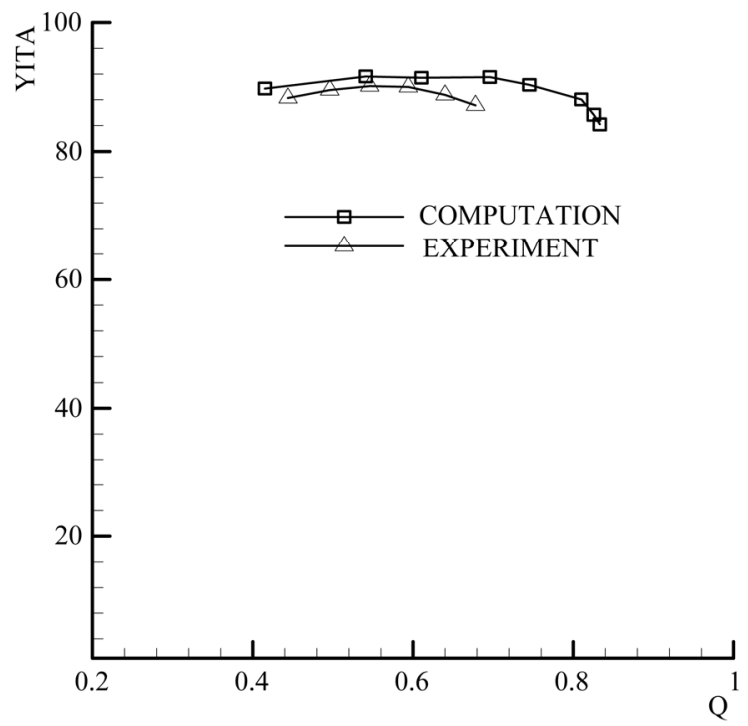

Figure 5. performance map of the AOCV centrifugal impeller.

the experiment, the y-axis is the impeller polytropic efficient $\eta=\frac{\kappa-1}{\kappa} \cdot \frac{\lg \left(P_{2}^{*} / P_{2}^{*}\right)}{\lg \left(T_{2}^{*} / T_{2}^{*}\right)}$, and the $\mathrm{x}$-axis is defined as the mass rate coefficient $Q=\left(Q_{i n} \times 10^{10}\right) /\left(D_{2}^{2} n b_{2}\right)$. The measured maximum polytropic efficiency is $90.125 \%$, correspondingly the mass rate coefficient is 0.546 ; the predicted maximum efficiency is $91.533 \%$ and the mass coefficient is 0.6960 . At the same time, the measured minimum polytropic efficiency is $87.125 \%$, correspondingly the mass rate coefficient is 0.678 , the predicted minimum efficiency is $84.209 \%$ and the mass coefficient is 0.833 . From the Figure 5 , the predicted efficiency is a little higher than that measured in experiment. The 3-D flow calculation with the time-marching method gives 
the broader work condition than the measurement.

\section{Conclusion}

A three-dimensional viscous code has been developed to solve Reynolds-averaged Navier-Stokes equations in finite volume form with time-marching method and the eddy viscous is obtained using the Baldwin-Lomax model. A prediction of the three-dimensional turbulent flow and the performance in the AOCV centrifugal impeller with a vaneless diffuser has been made for the compressor design and off-design condition. The predicted efficiency is a little higher than that tested in experiment. The presented results suggest that the present calculation code is able to determine the flow development in the impeller and the turbulence model in the centrifugal impeller should be improved.

\section{References}

[1] D. Eckardt, "Detailed Flow Investigations within a High Speed Centrifugal Compressor Impeller," Transaction of the ASME, Journal of Fluids Engineering, Vol. 98, No. 3390, 1976, pp. 390-402. doi:10.1115/1.3448334

[2] H. Krain and W. Hoffman, "Verification of an Impeller Design by Laser Measurements and 3D-Viscous Flow Calculations," ASME Paper, No. 89-GT-159, 1989.

[3] C. Hah and H. Krain, "Second Flows and Vertex Motion in a High-Efficiency Backswept Impeller at Design and off-Design Condition," ASME Journal of Turbomachinery, Vol. 112, No. 1, 1, 1990, pp. 7-13. doi:10.1115/1.29 27425

[4] R. Kunz and B. Laskshminarayana, "Navier-Stokes Investigation of a Transonic Centrifugal Compressor Stage Using an Algebraic Reynolds Stress Model," AIAA Paper, 1992, pp. 92-3311.

[5] W. N. Dawes, "A Numerical Analysis of the Three-Dimensional Viscous Flow in a Transonic Compressor Rotor and Comparison with Experiment," ASME Journal of Turbomachienry, Vol. 109, No. 1, 1987, pp. 83-109. doi: 10.1115/1.3262074 